\title{
Editorial note: Evaluating the effect of plant water availability on inner alpine coniferous trees based on sap flow measurements
}

\author{
Hans Pretzsch
}

Published online: 17 May 2014

(c) Springer-Verlag Berlin Heidelberg 2014

Due to an unfortunate technical mistake when handling the article "Evaluating the effect of plant water availability on inner alpine coniferous trees based on sap flow measurements" by M. Leo et al., R. Matyssek came into a double role as being one of the authors and as subject editor at the same time. Nevertheless, I guarantee that scientific standards and honesty have not been violated in any way.

Hans Pretzsch

Editor in Chief 